\title{
The effects of COVID-19 pandemic on pediatric neurosurgery practice and training in a developing country
}

\author{
Keyvan Tayebi Meybodi ${ }^{1} \cdot$ Zohreh Habibi $^{1}$ (D) Farideh Nejat $^{1}$
}

Received: 1 October 2020 / Accepted: 27 October 2020 / Published online: 1 November 2020

(C) Springer-Verlag GmbH Germany, part of Springer Nature 2020

\begin{abstract}
Purpose COVID-19 pandemic has influenced all aspects of societies, with the healthcare being the most affected field. All specialties including neurosurgery are involved, and due to resource limitations, the number of elective surgeries in subspecialized filed has substantially decreased. Herein, we report our practice experience in pediatric neurosurgery in a tertiary hospital during pandemic, and the effects of pandemic on educational issues.

Methods All the patients on whom any kind of neurosurgical operation was performed from March to June 2020 were retrospectively collected, and also from the same period in the previous year.

Results A total of 111 patients underwent surgery in this period. This figure was 159 patients during the same period in 2019. The total number of surgical cases reduced by $31 \%$ compared to the last year. While ventriculoperitoneal shunts and supratentorial tumor were more frequent, there was a considerable reduction in subspecialized educational surgeries like neural tube defects and craniosynostoses.

Conclusion CVID-19 pandemic changed all scopes of medical practice and training. Considering the limitation in the available resources, the number of educational cases may decrease in subspecialized disciplines like pediatric neurosurgery. If pandemic continues, alternative measures should be taken to compensate for the shortcoming in technical and practical training.
\end{abstract}

Keywords COVID pandemic $\cdot$ Pediatric neurosurgery $\cdot$ Practice $\cdot$ Training

\section{Introduction}

COVID-19 is a global problem. First described as pneumonia of unknown cause in Wuhan, China, in December 2019, it rapidly propagated in the globe as a disastrous cause of morbidity and mortality [12]. Up to now, more than 26 million of cases have been recorded all over the world with more than 850 thousand fatalities (https://www.worldometers.info/ coronavirus/ accessed: 2020/09/07).

Since COVID-19 outbreak in January 2020, and pandemic announcement by WHO on 11 March 2020, different papers and guidelines have been published about neurosurgery practice during COVID19 pandemics. All recommendations $[1,6,8]$ are in the same direction. Emergent and urgent cases

Zohreh Habibi

z-habibi@sina.tums.ac.ir

1 Department of Pediatric Neurosurgery, Children's Medical Center, Tehran University of Medical Sciences, Tehran 1419733151, Iran should be managed based on clinical indications, while protecting patients and medical staff according to local protocols. Elective surgeries should be postponed until the pandemic being kept under control. Once the pandemic is under control, elective surgeries should be scheduled, following hospital protocols for infection control.

Besides the implications of the problem in global health, COVID-19 has been associated with many educational problems in medical subspecialties. The resources have to be allocated to management of the patients with new viral infection and emergent problems; therefore, little will remain for many non-emergent educational cases throughout all the specialties of medicine and surgery. Hospitals have to be devoid of nonemergent cases which can be postponed to the time in which the resource restrictions are removed.

In Iran, the first cases of COVID-19 were registered in February 19, 2020 [10]. Since then, near 350 thousand of cases have been infected in the country and more than 22,000 patients have been dead (https://www.worldometers. info/coronavirus/country/iran/ accessed: 2020/09/07). Meanwhile, most medical centers in the country, either 
private or university hospitals, have been somehow involved with organizing and management of COVID-19 pandemic. $\mathrm{CMC}$ is a tertiary referral center for pediatric neurosurgery. It is a high-volume hospital with a vast spectrum of surgeries, which routinely provides a 2-month rotation for neurosurgery residents in Tehran University of Medical Science. This period is thoroughly determined in residency program for each given trainee as a part of PGY 3 rotations which cannot be replaced. Herein, we are to report our practice experience in the Department of Pediatric Neurosurgery in CMC hospital during COVID-19 pandemic, and the effects of pandemic on educational issues.

\section{Materials and Methods}

\section{Surgical cases}

In this case series, all the patients on whom any kind of neurosurgical operation was performed in CMC from March through June 2020 were retrospectively collected, and also from the same time period in the previous year. Also, the number of specialized elective surgeries, namely neural tube defects and craniosynostoses, observed by each resident during his/her pediatric neurosurgery rotation was recorded.

\section{Local protocol for COVID assessment and elective surgeries}

According to local guidelines in our hospital, no routine screening test was done preoperatively in asymptomatic patients till April 20. So, we sustained on personal protection and evaluating symptomatic patients, while handling just urgent and emergent cases. Since April 20, we started highly selected elective educational surgeries with preoperative COVID-19-PCR test and antibodies. No chest computed tomography (CT) was requested for asymptomatic cases, because of potential risk of radiation in pediatric population. Preoperative screening tests for elective surgeries stopped since June 1 according to local policies. After the second surge of the disease, restrictions were reestablished from the middle of June 2020 with further restrictions in performing elective cases.

\section{Statistics}

The descriptive results of surgical cases of pediatric neurosurgery department during a 3-month period compared with the last year are presented.

The study was approved by institutional ethics committee.

\section{Results}

One hundred eleven patients underwent surgery from March up to June 2020. This figure was 159 patients during the same period in 2019. The total number of surgical cases reduced by $31 \%$ compared to the last year. As shown in the Fig. 1, while ventriculoperitoneal shunts and supratentorial tumor surgeries were more operated on, there was a considerable reduction in subspecialized educational surgeries like operations on neural tube defects and craniosynostoses compared to previous year. Table 1 shows the comparative results of the number of each kind of surgery during 4 months in both years.

\section{Residents' experience with subspecialized surgeries during pandemic}

Among 4 neurosurgery residents who completed pediatric neurosurgery rotation between March and June 2020, two trainees could not be present in the field of any neural tube defect surgeries and one other could not observe any myelomeningocele surgery. Only one out four residents has the opportunity to observe both open and closed spinal dysraphism surgeries. None of the residents could observe any kind of open craniosynostosis surgeries. Three residents were present in the field of endoscopic suturectomy for metopic and coronal craniosynostosis, and the other trainee had no chance to see any endoscopic craniosynostosis surgery. Endoscopic sagittal suturectomy was not observed by any of the trainees during this period.

\section{Discussion}

This is a study on change in the type of pediatric neurosurgery practice and training during pandemic. Indeed, with the outbreak of COVID-19, the pattern and rate of surgeries in our tertiary center were substantially changed. During the first month of outbreak, we were overwhelmed with a great number of urgent/emergent cases of brain tumors and acute hydrocephalus which were referred from other centers in the country. Besides, as shown in Fig. 1, cases of pediatric neurosurgery that our trainees could observe in a subspecialized pediatric ward, including craniosynostosis, closed NTD, myelomeningocele, encephalocele, and seizure surgery have decreased due to limited resource and current policies induced by pandemic.

\section{COVID-19 and pediatric neurosurgery practice}

COVID-19 keeps living with us. In many countries, after relative freedom of restrictions, the infection resurged. Up to our knowledge, there is no absolute treatment or vaccine against the virus. It seems that until development of a vaccine or specific therapy, practice of medicine and especially 


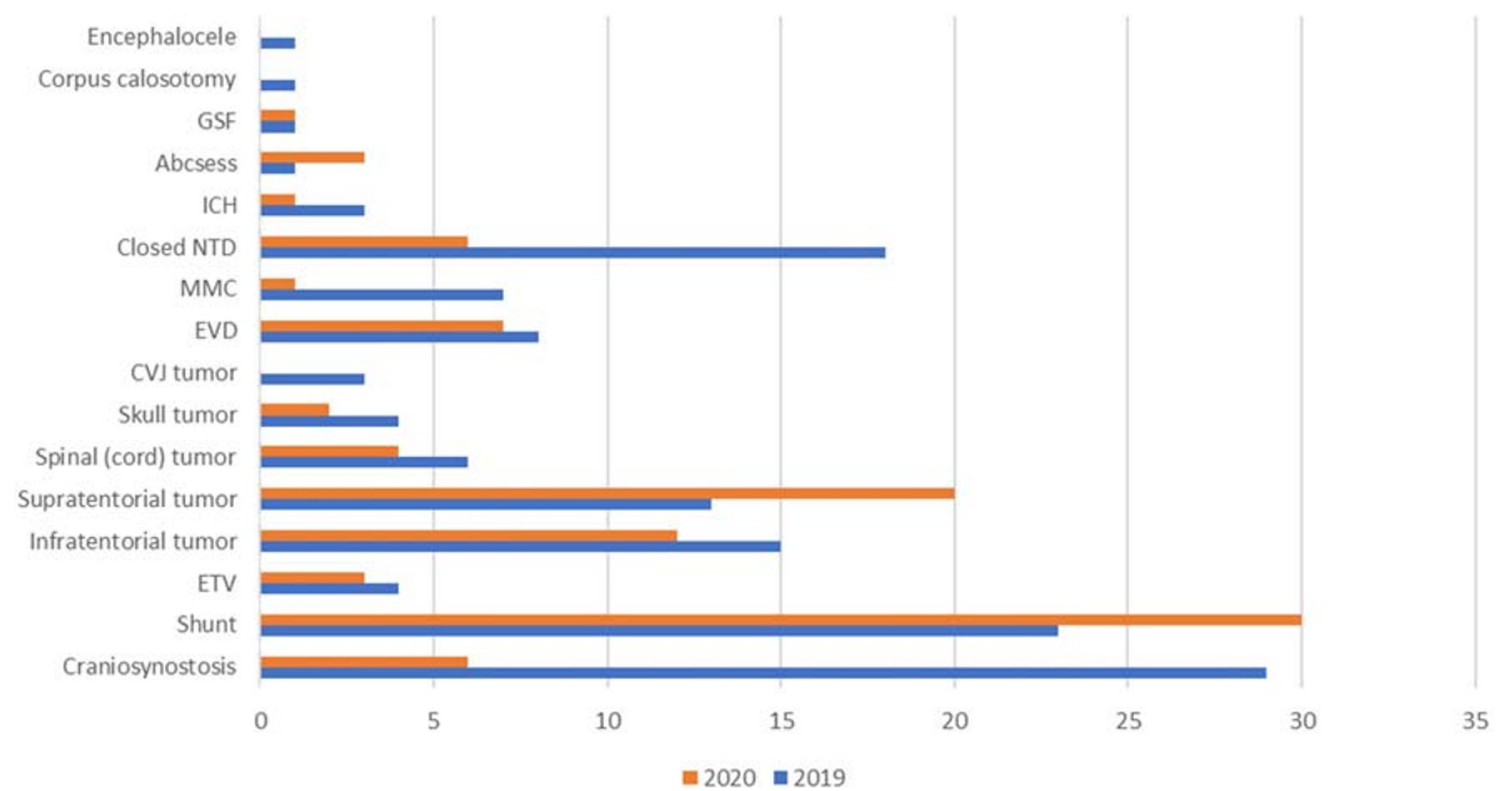

Fig. 1 The number of each kind of surgery performed through March to June in 2019 and 2020. The number of surgeries performed on closed neural tube defects and craniosynostoses was reduced drastically in 2020

performing surgeries have to meet the policies and available resources, along with the surgical indications.

There are two axioms to which we have to adhere in our practice, as others: (1) urgent/emergent cases should be

Table 1 The comparative results of the number of surgeries during March to June in both years

\begin{tabular}{|c|c|c|}
\hline Surgery & 2019 (March to June) & 2020 (March to June) \\
\hline Infratentorial tumor & 15 & 12 \\
\hline Supratentorial tumor & 13 & 20 \\
\hline Spinal (cord) tumor & 6 & 4 \\
\hline Skull tumor & 4 & 2 \\
\hline CVJ tumor & 3 & 0 \\
\hline Shunt & 23 & 30 \\
\hline ETV & 4 & 3 \\
\hline EVD & 8 & 7 \\
\hline Craniosynostosis & 29 & 6 \\
\hline Closed NTD & 18 & 6 \\
\hline Myelomeningocele & 7 & 1 \\
\hline $\mathrm{ICH}$ & 3 & 1 \\
\hline Abscess & 1 & 3 \\
\hline GSF & 1 & 1 \\
\hline Corpus callosotomy & 1 & 0 \\
\hline Encephalocele & 1 & 0 \\
\hline Minor procedures* & 22 & 15 \\
\hline Total & 159 & 111 \\
\hline
\end{tabular}

$C V J$, craniovertebral junction; ETV, endoscopic third ventriculostomy; $E V D$, external ventricular drainage; $N T D$, neural tube defect; $I C H$, intracerebral hemorrhage; $G S F$, growing skull fracture

*Lumbar puncture, fontanelle tap, ventricular shunt tap managed anyway [4], and (2) maximum protection of patients and staff is of great importance [3]. Decision making about testing asymptomatic patients should be customized base on many factors related to the patients, society, medical centers, and the type of surgery. In a country with restricted resources, testing all the patients may not be cost-effective; therefore, performing the diagnostic test for the symptomatic patients is the standard.

In this series the rate of surgeries showed 31\% decrease compared to the last year. This is in line with others' practice. For instance, Deora et al. have highlighted the reduction in operation room case volume, i.e., elective cases, during the pandemic [2]. But sometimes, the procrastination of surgery may harbor a negative consequence on the patient, risks of the surgical operation, and also the type of operation itself. For example, some of the endoscopic craniosynostosis surgeries cannot be postponed to after 6 months of age, after which the type of surgery has to be changed to a more challenging open craniotomy. Another important issue is the lost revenue due to cancelation of elective cases. Although we did not study this issue in our series, it seems that reduced hospital income from canceled surgeries may have dire consequences on hospital resources [7]. The remainder of the resources should be allocated to COVID control; henceforth, the vicious cycle of cancelation of some elective surgeries due to limited available resources and reduced subspecialized training cases will continue.

\section{COVID-19 and pediatric neurosurgery training}

Education of residents and fellows remains a crucial issue in the era of COVID-19. Although e-learning and virtual 
education may play an important role in the education of trainees (undergraduate and postgraduate), this may not make sense in the surgical realm, since e-learning cannot compensate for a manual skill which has to be learned best by interaction with a real patient [9]. Moreover, being involved in the preoperative and postoperative management of patients is of great value in training surgical practice and cannot be provided via virtual fields. Because the numbers of elective cases are reduced after the pandemic, trainees may feel defects in their training program especially with subspecialized cases. Local policies for social distancing or lockdown may further complicate the mentioned situation. In our series, surgeries for neural tube defects and craniosynostosis patients were canceled pending a more favorable condition. Our hospital is a tertiary referral pediatric center, which provides a $2-$ month rotation for PGY 3 neurological surgery residents to be involved in pediatric neurosurgery. According to Fig. 1, most of surgeries performed in our department during pandemic were tumors and hydrocephalus which are frequently done in the other neurosurgery departments of the university, while neural tube defects and craniosynostoses are mainly performed in CMC. With this short duration of rotation, and reduced total surgical cases and, especially, greatly reduced burden of subspecialized pediatric cases, there will be a significant deficiency in the pediatric neurosurgical residency education. Indeed, residents may have no more chance to be involved in the field of such surgeries during their training.

What do we have to do for impaired residency education in surgical skill training and also surgical skill maintenance? According to Lewis et al., there is no good-quality replacement for operative experience, even in developed countries [9]. However, there may be some mid-quality options:

1. Televised live surgery using microscope or endoscope $[3$, 5]

2. TV camera embedded in the surgical lights [3]

3. Simulation-based surgery and artificial intelligence technologies $[9,11]$

None of the above may make sense for training residents in a low-resource developing country. Indeed, there may be additional problems in conducting distance learning and virtual training due to challenges for providing high-speed internet, online platforms, technical facilities, and access to net by trainees. Hence, the answer remains elusive. May time answer this question, and may COVID-19 pandemic open the way in the field of virtual education for surgical training.

\section{Conclusion}

CVID-19 pandemic has changed all scopes of medical practice and training. In the lack of unified protocol and considering the limitation in the available resources, the number of educational cases may decrease in subspecialized disciplines like pediatric neurosurgery. If pandemic continues, alternative measures should be taken to compensate for shortcoming in practical and technical training.

\section{Compliance with ethical standards}

The study was approved by institutional ethics committee.

Conflict of interest The authors report no conflict of interest concerning the materials or methods used in this study or the findings specified in this paper.

\section{References}

1. Burke JF, Chan AK, Mummaneni V, Chou D, Lobo EP, Berger MS, Theodosopoulos PV, Mummaneni PV (2020) Letter: The coronavirus disease 2019 global pandemic: a neurosurgical treatment algorithm. Neurosurgery. 87(1):E50-E56. https://doi.org/10.1093/ neuros/nyaal

2. Deora H, Mishra S, Tripathi M, Garg K, Tandon V, Borkar S, Varshney N, Raut R, Chaurasia B, Chandra PS, Kale SS (2020) Adapting neurosurgery practice during the COVID-19 pandemic in the Indian subcontinent. World Neurosurg. S1878-8750(20): 31549-31547. https://doi.org/10.1016/j.wneu.2020.07.038

3. Fernandes Cabral DT, Alan N, Agarwal N, Lunsford LD, Monaco EA 3rd (2020) Coronavirus disease 2019 (COVID-19) and neurosurgery residency action plan: an institutional experience from the United States. World Neurosurg. S1878-8750(20):31601-31606. https://doi.org/10.1016/j.wneu.2020.07.080

4. Ghogawala Z, Kurpad S, Falavigna A, Groff MW, Sciubba DM, Wu JC, Park P, Berven S, Hoh DJ, Bisson EF, Steinmetz MP, Wang MC, Chou D, Sansur CA, Smith JS, Tumialán LM (2020) Editorial. COVID-19 and spinal surgery. J Neurosurg Spin 17:1-3. https://doi.org/10.3171/2020.4.SPINE20468

5. Jack MM, Gattozzi DA, Camarata PJ, Shah KJ (2020) Livestreaming surgery for medical student education - educational solutions in neurosurgery during the COVID-19 pandemic. J Surg Educ. [ S1931-7204(20):30239-30237. https://doi.org/10.1016/j. jsurg.2020.07.005

6. Jean WC, Ironside NT, Sack KD, Felbaum DR, Syed HR (2020) The impact of COVID-19 on neurosurgeons and the strategy for triaging non-emergent operations: a global neurosurgery study. Acta Neurochir 162(6):1229-1240

7. Khalafallah AM, Jimenez AE, Lee RP, Weingart JD, Theodore N, Cohen AR, Tamargo RJ, Huang J, Brem H, Mukherjee D (2020) Impact of COVID-19 on an Academic Neurosurgery Department: The Johns Hopkins experience. World Neurosurg. [ 139:e877e884. https://doi.org/10.1016/j.wneu.2020.05.167

8. Kondziolka D, Couldwell WT, Rutka JT (2020) Introduction. On pandemics: the impact of COVID-19 on the practice of neurosurgery. J Neurosurg 10:1-2. https://doi.org/10.3171/2020.3. JNS201007

9. Lewis CT, Zeineddine HA, Esquenazi Y (2020) Challenges of neurosurgery education during the coronavirus disease 2019 (COVID19) pandemic: a U.S. perspective. World Neurosurg. [ 138:545547. https://doi.org/10.1016/j.wneu.2020.04.179

10. Raoofi A, Takian A, Akbari Sari A, Olyaeemanesh A, Haghighi H, Aarabi M (2020) COVID-19 pandemic and comparative health policy learning in Iran. Arch Iran Med 23(4):220-234 
11. Scullen T, Mathkour M, Maulucci CM, Dumont AS, Bui CJ, Keen JR (2020) Letter to the Editor Impact of the COVID-19 pandemic on neurosurgical residency training in New Orleans. World Neurosurg. [ 139:718-719. https://doi.org/10.1016/j.wneu.2020. 04.208

12. Zhu N, Zhang D, Wang W, Li X, Yang B, Song J, Zhao X, Huang B, Shi W, Lu R, Niu P, Zhan F, Ma X, Wang D, Xu W, Wu G, Gao
GF, Tan W, China Novel Coronavirus Investigating and Research Team (2020) A novel coronavirus from patients with pneumonia in China, 2019. N Engl J Med 382(8):727-733

Publisher's note Springer Nature remains neutral with regard to jurisdictional claims in published maps and institutional affiliations. 